\title{
Solitary Pituitary Metastasis of Advanced Breast Cancer Treated with Anti-Human Epidermal Growth Factor Receptor 2 Drug
}

\author{
Jin Won Jang ${ }^{1,2}$, Kyung Ae Lee ${ }^{1,2}$, Won Sik Jung ${ }^{1,2}$, Ja Yeon Lee ${ }^{1,2}$, Sunghoon Choi ${ }^{1,2}$, Yoon Chae Lee ${ }^{1,2}$ \\ ${ }^{1}$ Institute of Clinical Medicine, Chonbuk National University Hospital; ${ }^{2}$ Department of Internal Medicine, Chonbuk National University College of Medicine, \\ Jeonju, Korea
}

\begin{abstract}
Metastasis to the pituitary gland from systemic cancer is a rare condition. The breast and lung are the most common sites of primary tumor metastasis. Most often, they occur in the setting of widespread metastatic disease, which most frequently occurs in elderly patients. However, an increase in the incidence of solitary pituitary metastasis of breast cancer as the first recurrence has been reported. Diabetes insipidus is the most frequent symptom at presentation, and visual field defects or cranial nerve deficits are common symptoms of pituitary metastasis. Unlikely these symptoms, deficiencies of anterior pituitary hormones may only become evident in critical situation because symptoms are of an insidious onset and sometimes nonspecific. We report here on a rare case of solitary pituitary metastasis from breast cancer presenting as hyponatremia without other symptoms.
\end{abstract}

Keywords: Breast neoplasms; Metastasis; Pituitary gland; Hyponatremia

\section{INTRODUCTION}

Recently, following the introduction of adjuvant therapy with the anti-human epidermal growth factor receptor 2 (HER2) therapy trastuzumab (Herceptin) in the treatment of breast cancer, an increase in the frequency of central nervous system (CNS) metastasis as the site of first recurrence has been reported [1]. However, it is difficult to diagnose pituitary metastases, as the symptoms are nonspecific and the radiological differences from primary tumors are trivial [2]. In this report, we describe a rare case of a solitary metastatic breast cancer to the pituitary, presenting as hyponatremia without other common symptoms.

\section{CASE REPORT}

A 59-year-old woman was brought to the emergency department because of nausea and dizziness. Three years prior, the patient was diagnosed as having breast cancer and underwent modified radical mastectomy and subsequent adjuvant chemotherapy. Because the tumor expressed a novel HER2 mutation, the patient received trastuzumab chemotherapy, which was proven beneficial to patients. However, 18 months prior to presentation, solitary brain metastasis in the frontal cortex, without any other metastasis, was detected on brain magnetic resonance imaging (MRI) (Fig. 1). After tumor mass removal, systemic chemotherapy was initiated. Then, serial brain MRI was performed at 3-month intervals and showed no recurring mass in the operation site. However, the patient was brought to our emergency department with a chief compliant of dizziness, nausea, and poor oral intake. Laboratory examination results showed hyponatremia ( $\mathrm{Na}$ level, $122 \mathrm{mmol} / \mathrm{L}$; reference range, 135 to $150 \mathrm{mmol} / \mathrm{L}$ ). To determine the cause of the hyponatremia, a hormonal workup was performed. The morning levels of cortisol ( $0.4 \mu \mathrm{g} / \mathrm{dL}$; reference value $>10 \mu \mathrm{g} / \mathrm{dL})$, adrenocorticotropic hormone $(10.8 \mathrm{pg} / \mathrm{mL})$, free T4 (6.09 pmol/L; reference range, 11.5 to $22.7 \mathrm{pmol} / \mathrm{L})$, thyroid stimulating hormone $(0.486$ $\mathrm{mIU} / \mathrm{mL}$; reference range, 0.55 to $4.78 \mathrm{mIU} / \mathrm{mL}$ ), insulin-like growth factor type 1 ( $28 \mathrm{ng} / \mathrm{mL}$; reference range, 55 to $225 \mathrm{ng} / \mathrm{mL}$ ), growth hormone $(0.14 \mathrm{ng} / \mathrm{mL}$; reference value $>1.0 \mathrm{ng} / \mathrm{mL})$, follicle
Correspondence to: Kyung Ae Lee

Division of Endocrinology and Metabolism, Department of Internal Medicine, Chonbuk National University Hospital, Chonbuk National University Medical School, 20 Geonji-ro, Deokjin-gu, Jeonju 54907, Korea

Tel: +82-63-250-2749, Fax: +82-63-254-1609, E-mail: kaleey@jbnu.ac.kr

Received: Jun. 10, 2015 / Accepted after revision: Aug. 28, 2015
(C) 2015 Soonchunhyang Medical Research Institute This is an Open Access article distributed under the terms of the Creative Commons Attribution Non-Commercial License (http://creativecommons.org/licenses/by-nc/3.0/). 
stimulating hormone $(5.5 \mathrm{mIU} / \mathrm{mL})$, luteinizing hormone $(<0.07$ $\mathrm{mIU} / \mathrm{mL})$, estradiol $(<5.0 \mathrm{pg} / \mathrm{mL})$, and prolactin $(9.53 \mathrm{ng} / \mathrm{mL}$; reference range, 4.79 to $23.3 \mathrm{ng} / \mathrm{mL}$ ). All anterior pituitary hormones

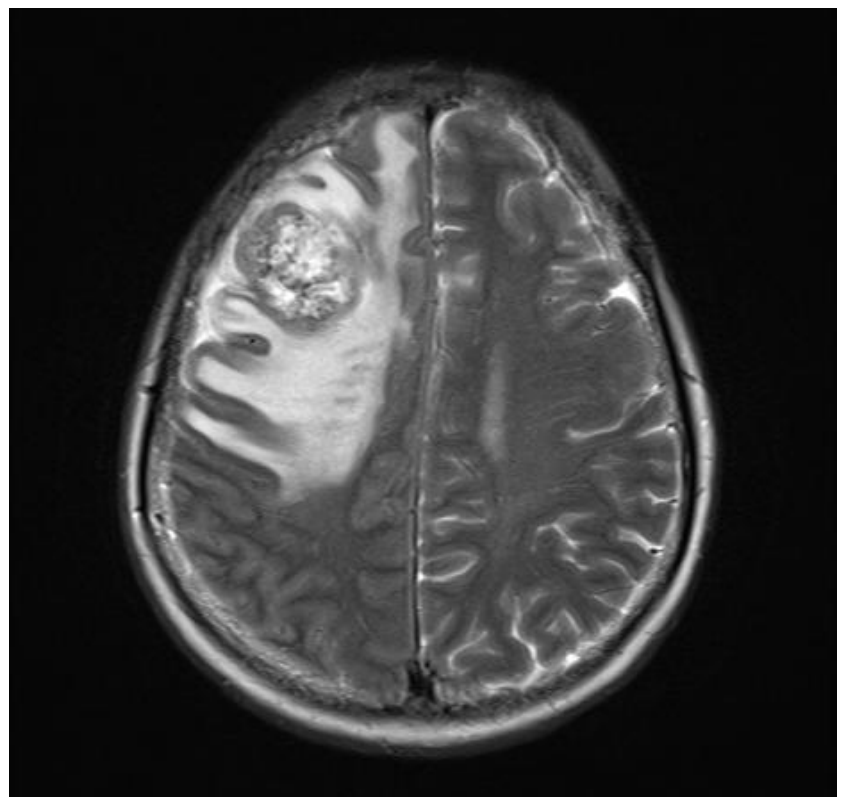

Fig. 1. Brain magnetic resonance imaging showing a $3.5 \times 3.5 \times 3.5-\mathrm{cm}$ heterogeneous enhancing mass with cystic necrosis in the right frontal lobe. Severe vasogenic edema is seen around the tumor mass.

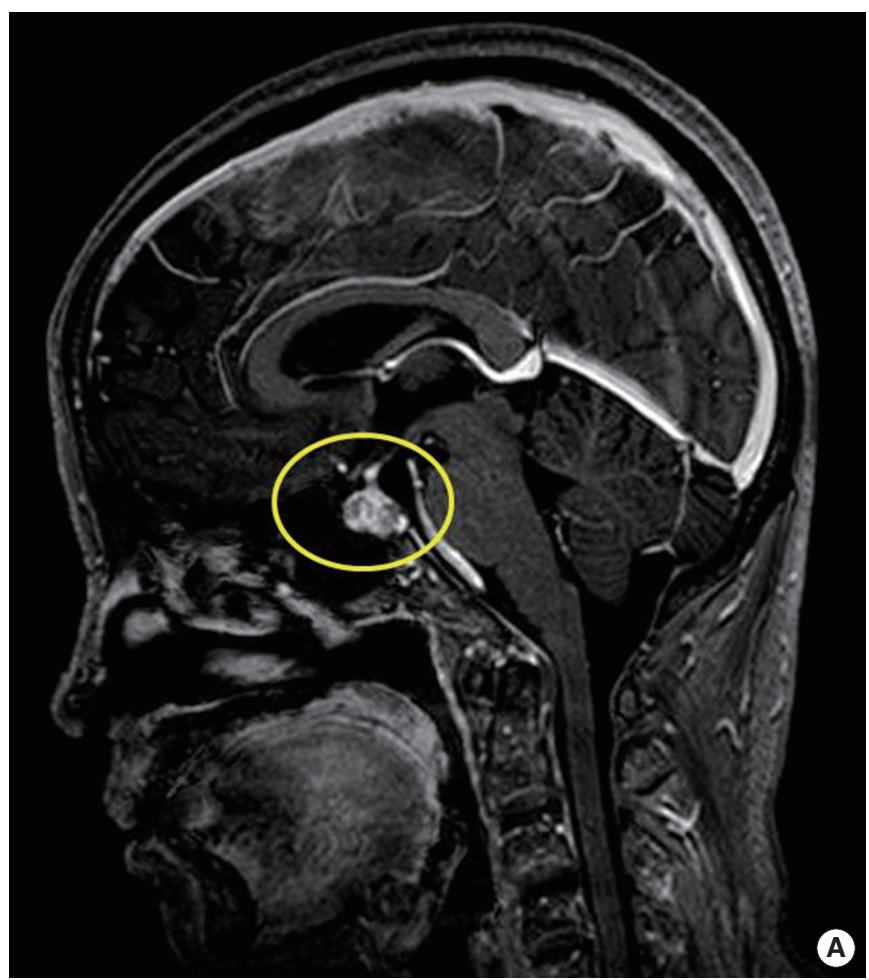

were lower than their normal limits. However, the patient had no visual symptoms and her urine amount was within the reference range. Brain MRI was performed and showed pituitary gland enlargement with heterogeneous enhancement that was indicative of pituitary metastasis (Fig. 2). We reviewed previous brain MRI findings and found that the pituitary gland had progressively, insidiously enlarged during the 10-month period. Dynamic sellar MRI was not performed in this patient. The thyroid function and gonadotropin levels had been normal until 8 months ago. Hormonal replacement with glucocorticoid and thyroxine was started, and both hyponatremia and associated symptoms were markedly improved. Gamma knife surgery for the pituitary metastasis was performed. A follow-up MRI revealed that the metastatic lesion had markedly decreased in size. The patient then received oral chemotherapy with a novel tyrosine kinase inhibitor and concurrent hormone replacement. At the last follow-up examination in the outpatient clinic, her general condition was found to be relatively good.

\section{DISCUSSION}

The pituitary gland is a rare site of metastasis of all neoplasms, accounting for only $1 \%$ of pituitary lesions [3]. Breast and lung can-

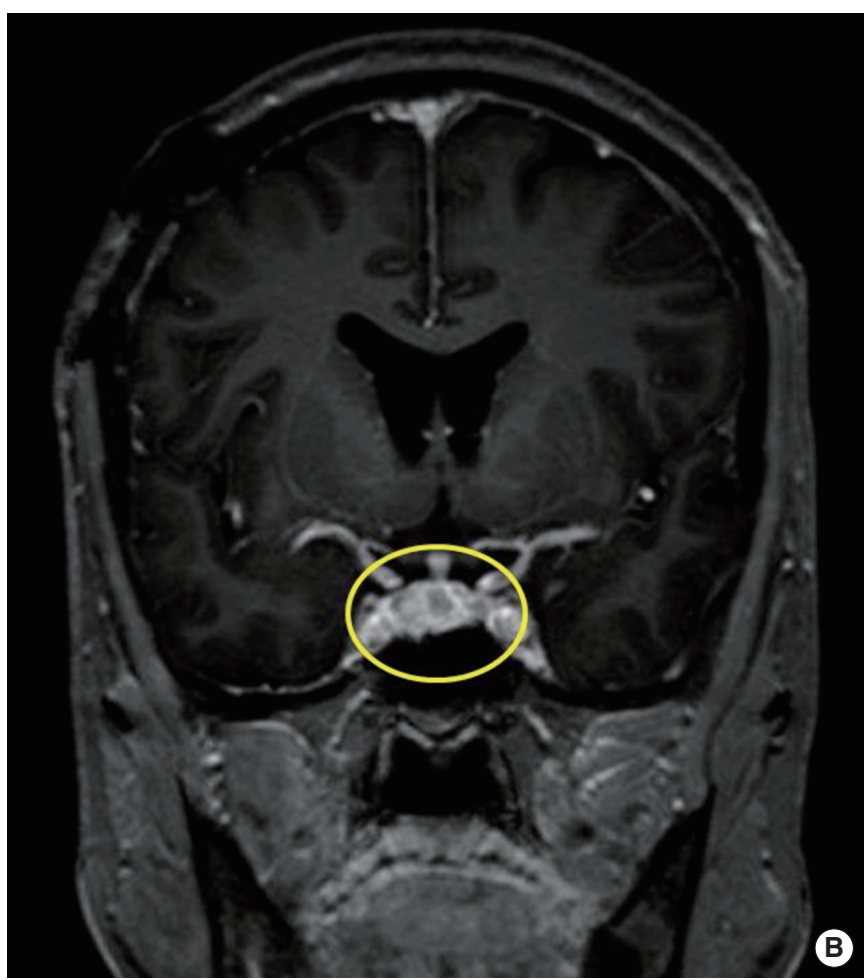

Fig. 2. Brain magnetic resonance imaging showing pituitary gland enlargement with heterogeneous enhancement that was indicative of pituitary metastasis (yellow circle). (A) Sagittal view and (B) coronal view. 
Jang JW, et al. • Hyponatremia without Other Symptoms by Solitary Pituitary Metastasis of Breast Cancer

cers are the most common primary neoplasms that metastasize to the pituitary gland. Most often, they occur in the setting of widespread metastatic disease, which most frequently occurs in elderly patients [4]. However, since the introduction of the novel targeted therapy with trastuzumab (anti-HER2 therapy) for the treatment of breast cancer, approximately one-third of patients with metastatic HER2-positive breast cancer will develop CNS metastases including pituitary metastasis [1]. However, solitary pituitary metastasis from breast cancer is extremely rare. There have been three other reports of isolated pituitary metastasis [5-7]. In Korea, only one report describes a solitary pituitary metastasis diagnosed after the end of adjuvant trastuzumab therapy [7]. It has been suggested that trastuzumab may not be active in the CNS, which could be a potential sanctuary site for disease progression [5]. In the case of solitary pituitary metastasis, differential diagnosis between pituitary metastasis and primary pituitary lesions such as adenoma, especially in the early stage of metastasis, is challenging [8]. Majority of pituitary metastasis are clinically silent and too small to cause radiological changes [4]. Even when symptomatic, radiological evaluation generally has not been useful in distinguishing from pituitary adenomas, unless other metastatic brain lesions coexist. But, rapid increase of a sellar mass with aggressive infiltration of adjacent tissues should raise suspicion of metastatic tumor $[9,10]$. And posterior lobe involvement is more common in the case of pituitary metastasis. Diabetes insipidus (DI) is the most frequent symptom at presentation, and visual field defects or cranial nerve deficits are common symptoms of pituitary metastasis [2]. By contrast, symptomatic anterior pituitary hormone deficiencies have been less frequently described at presentation. Among these symptoms, pituitary adenoma rarely presents with DI. In contrast to central DI, clinicians easily overlook a diagnosis of anterior hypopituitarism because of insidious onset and slow progression of hormone deficiency. In our patient, after metastasectomy for frontal lobe metastasis, conventional brain MRI was performed regularly. However, pituitary metastasis was detected only after the patient was brought to the emergency department because of symptoms of hyponatremia. Delayed diagnosis of pituitary metastasis may lead to serious conditions such as pituitary apoplexy or adrenal crisis, especially in cancer patients with poor general conditions. With the development of novel therapeutic agents, the number of breast cancer survivors has increased worldwide. Therefore, the incidence of pituitary metastasis of breast cancer might have increased concurrently. Clinicians should consider the possibility of pituitary metastasis in these patients. In patients with symptoms indicative of hormone deficiency or those with suspicious conventional MRI results, pituitary hormone evaluation and dynamic sellar MRI should be considered. Early diagnosis and proper management for pituitary metastasis in cancer patients can lead to decreased morbidities associated with hormone deficiencies.

\section{REFERENCES}

1. Olson EM, Abdel-Rasoul M, Maly J, Wu CS, Lin NU, Shapiro CL. Incidence and risk of central nervous system metastases as site of first recurrence in patients with HER2-positive breast cancer treated with adjuvant trastuzumab. Ann Oncol 2013;24:1526-33.

2. Komninos J, Vlassopoulou V, Protopapa D, Korfias S, Kontogeorgos G, Sakas DE, et al. Tumors metastatic to the pituitary gland: case report and literature review. J Clin Endocrinol Metab 2004;89:574-80.

3. Fassett DR, Couldwell WT. Metastases to the pituitary gland. Neurosurg Focus 2004;16:E8.

4. Teears RJ, Silverman EM. Clinicopathologic review of 88 cases of carcinoma metastatic to the putuitary gland. Cancer 1975;36:216-20.

5. Kam J, Kam J, Mann GB, Phillips C, Wentworth JM, King J, et al. Solitary pituitary metastasis from HER2-positive breast cancer. Asia Pac J Clin Oncol 2015 Apr 14 [Epub]. http://dx.doi.org/10.1111/ajco.12353.

6. Azambuja E, Piccart MJ, Awada A. Long-term survival in pituitary metastasis from breast cancer. Breast 2006;15:446-7.

7. Park Y, Kim H, Kim EH, Suh CO, Lee S. Effective treatment of solitary pituitary metastasis with panhypopituitarism in HER2-positive breast cancer by lapatinib. Cancer Res Treat 2015 Feb 16 [Epub]. http://dx.doi. org/10.4143/crt.2014.165.

8. Marin F, Kovacs KT, Scheithauer BW, Young WF Jr. The pituitary gland in patients with breast carcinoma: a histologic and immunocytochemical study of 125 cases. Mayo Clin Proc 1992;67:949-56.

9. Schubiger O, Haller D. Metastases to the pituitary--hypothalamic axis: an MR study of 7 symptomatic patients. Neuroradiology 1992;34:131-4.

10. Freda PU, Wardlaw SL, Post KD. Unusual causes of sellar/parasellar masses in a large transsphenoidal surgical series. J Clin Endocrinol Metab 1996; 81:3455-9. 